\title{
Shape, size and assembly control of PbTe nanocrystals
}

\author{
Taleb Mokari ${ }^{1}$, Minjuan Zhang ${ }^{2}$, Peidong Yang ${ }^{1,}$ \\ ${ }^{1}$ Department of Chemistry, University of California, Berkeley \\ Berkeley, CA 94720 \\ ${ }^{2}$ Materials Research Department, Toyota Technical Center, Toyota Motor Engineering \& \\ Manufacturing North America (TEMA) Inc. \\ 2350 Green Road, Ann Arbor, MI 48105
}

\section{Supporting Information}

\section{Experimental Section}

Chemicals. Amorphous tellurium powder (Aldrich, 99,999\%), lead acetate trihydrate (Fisher Scientific Co.), Tri-n-octylphosphine (TOP), Hexadecylamine (HDA) and Dodecylamine (DDA) were purchased from Aldrich. n-tetra- decylphosphonic acid (TDPA), Hexylphosphonic acid (HPA) and Octadecylphosphonic acid (ODPA) (Alfa, 97\%) were used as purchased without further purification. Diphenyl ether (DPE) and oleic acid were purchased from Aldrich. All syntheses were performed under Ar using a Schlenck line.

Synthesis of PbTe nanocrystals with various shapes. $0.4 \mathrm{~g}$ of lead acetate trihydrate and $1 \mathrm{~mL}$ of oleic acid were dissolved in $10 \mathrm{~mL}$ of DPE. This solution was heated to 150 ${ }^{\circ} \mathrm{C}$ for at 30 min under Ar to form a lead oleate complex. After 30 min the solution was cooled to room temperature and mixed with the Te solution (the amount of the Te depends on the ratio) in $4 \mathrm{ml}$ TOP. The Te solution was added slowly to prevent $\mathrm{PbTe}$ nucleation. This PbTe solution was injected under vigorous stirring into a hot $\left(250^{\circ} \mathrm{C}\right)$ growth solution containing $0.3 \mathrm{~g}$ of HDA dissolved in $15 \mathrm{~mL}$ of DPE. After the injection the temperature dropped to $170-180{ }^{\circ} \mathrm{C}$ and was maintained at this temperature for $3-4$ min, then the reaction mixture was cooled to room temperature using a water bath. The 
crude solution was mixed with an equal volume of Toluene and Methanol, and the nanoparticles were precipitated by centrifugation at $6000 \mathrm{rpm}$ for $5 \mathrm{~min}$. The product (precipitate) was redispersed in chloroform or toluene for further characterization.

Synthesis of PbTe cuboctahedral nanoparticles with different sizes. $0.38 \mathrm{~g}$ of lead acetate trihydrate and $1 \mathrm{~mL}$ of oleic acid were dissolved in $5 \mathrm{~mL}$ of TOP. This solution was heated to $150{ }^{\circ} \mathrm{C}$ for at least $45 \mathrm{~min}$ under Ar to form a lead oleate complex. After 45 min the solution was cooled to $50{ }^{\circ} \mathrm{C}$, and mixed with the Te solution $(0.04 \mathrm{~g}$ of Te in 2 ml TOP). The Te solution was added slowly to prevent PbTe nucleation. This PbTe solution was injected under vigorous stirring into a hot $\left(250^{\circ} \mathrm{C}\right)$ growth solution containing $0.1 \mathrm{~g}$ of TDPA (or equivalent mole amount of HPA/TDPA) dissolved in $5 \mathrm{~mL}$ of TOP (the growth solution was purified by heating to $180^{\circ} \mathrm{C}$ ). After the injection the temperature dropped to $150-160{ }^{\circ} \mathrm{C}$ and was maintained at this temperature for $\sim 2-8$ min (depends on the desired size), then the separation of the particles follow the same recipe as mention before.

Langmuir-Blodgett (LB) assembly: The precipitated PbTe nanocrystals were redispersed in $5 \mathrm{~mL}$ of chloroform with sonication. LB experiments were done with deionized water on a LB trough (Nima Technology, M611) at room temperature. The surface pressure was monitored with a Wilhelmy plate, and was zero before spreading the particles. The chloroform solution of PbTe nanocrystals was slowly spread on the water surface of the trough. The resulting surface layer was compressed by moving the mobile barrier at a rate of $20 \mathrm{~cm}^{2} / \mathrm{min}$. 


\section{Sample characterization}

The structure and composition of the nanocrystals were investigated by Transmission Electron Microscopy (TEM) (Technai 12) at 100 kV, High Resolution TEM (HRTEM) (Philips, CM200) at 200 kV, scanning Energy-Dispersive X-ray spectroscopy (EDX) and Electron Energy Loss Spectroscopy (EELS). The X-Ray Diffraction (XRD) patterns were taken using $\mathrm{Cu} \mathrm{K} \alpha$ radiation (1.790 $\AA$ ) and a general area detector (GADDS, Bruker). Samples were prepared by depositing precipitated powder onto a quartz plate. 


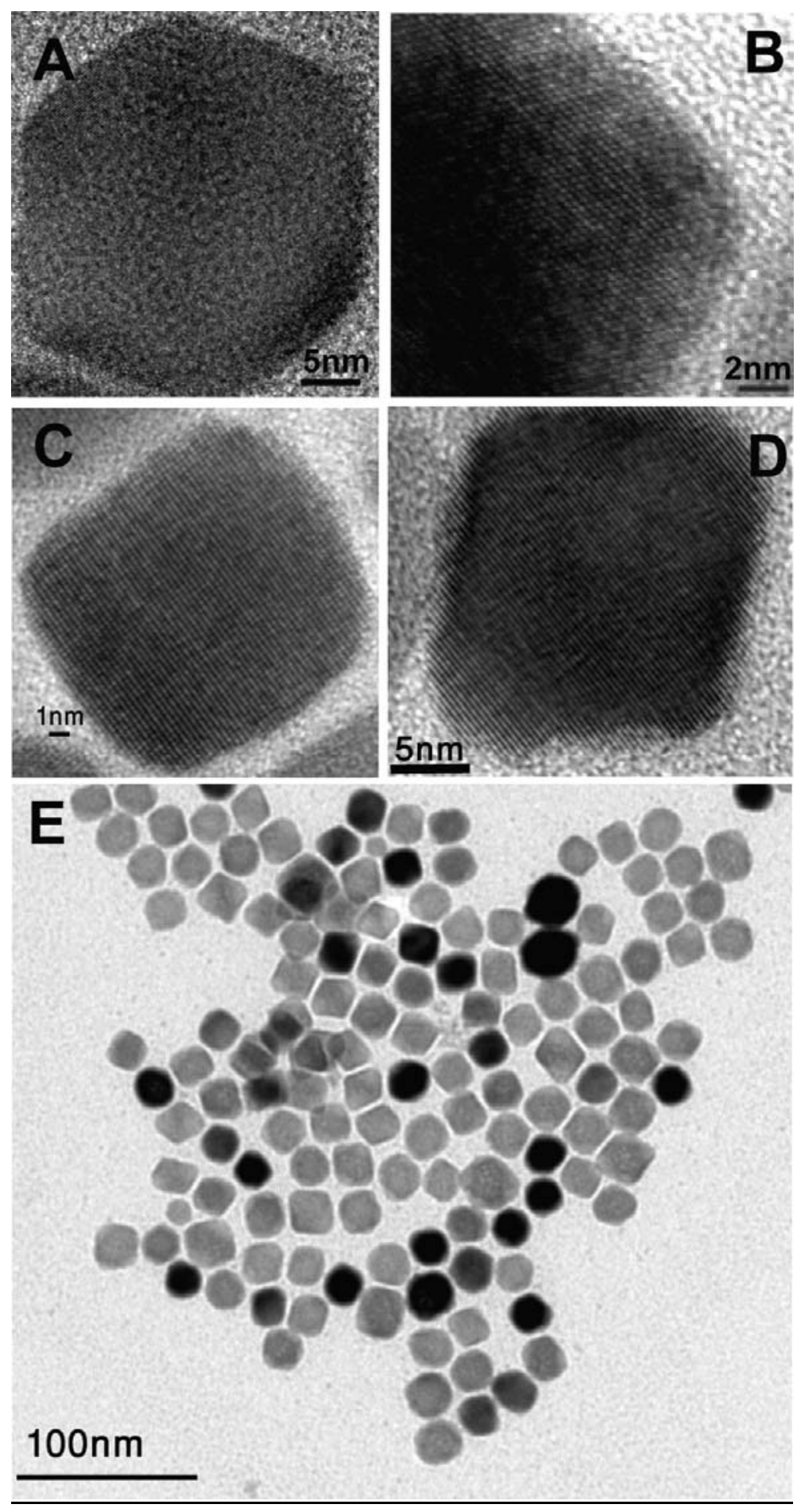

Fig. S1: HRTEM images of PbTe nanoparticles with different shapes, (A) Cuboctahedra, (B) A high magnification TEM image of cuboctahedral particle, (C) Cube, (D) HRTEM of octahedra and (E) low magnification TEM image of octahedral particles. 


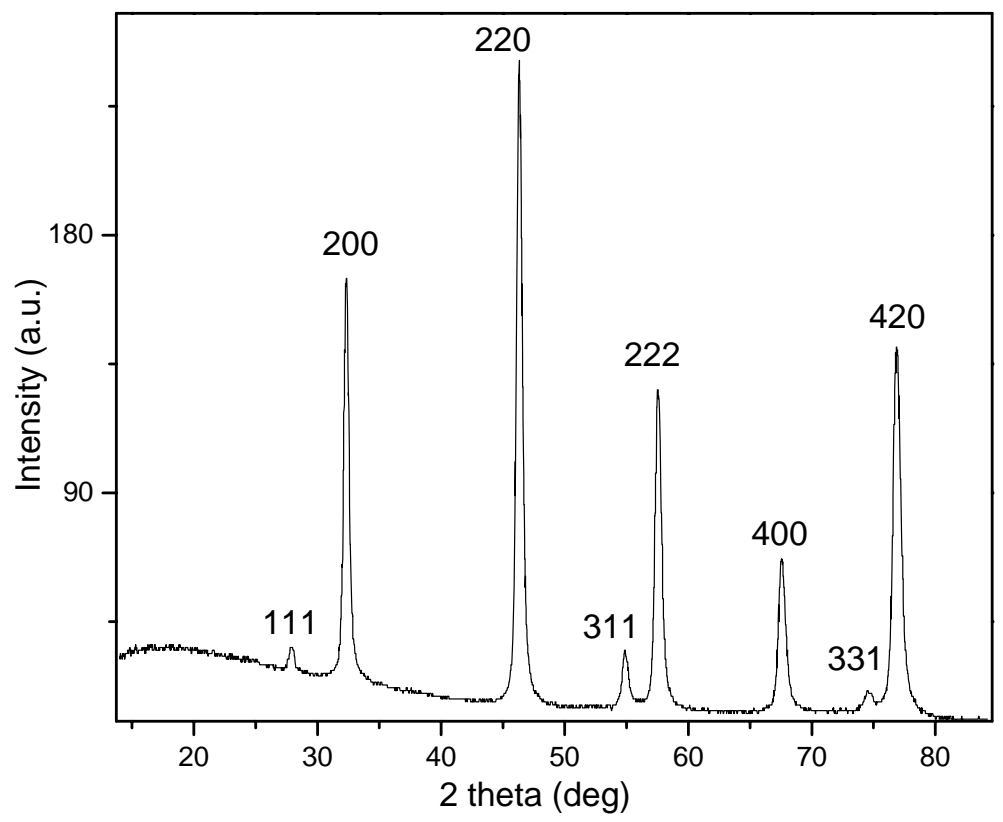

Fig. S2: The powder X-ray diffraction (XRD) measurement on PbTe nanocrystals agrees perfectly with the PbTe bulk. 


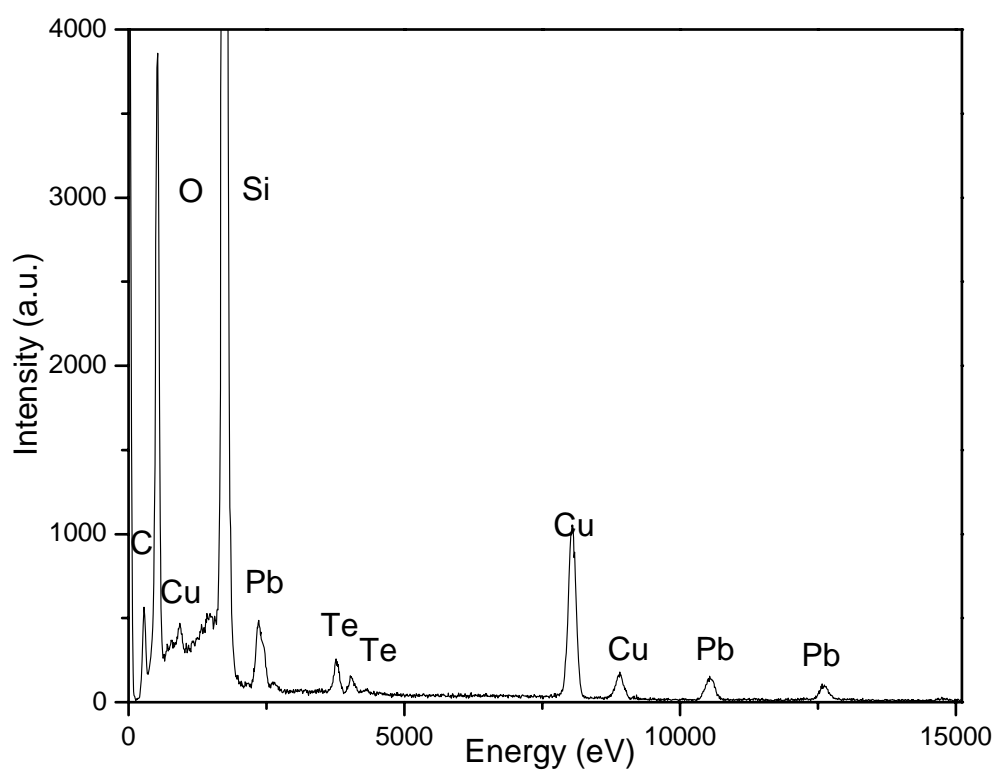

Fig. S3: The EDS spectrum of single PbTe cuboctahedral particle that is shown in Figure $1 B$. 

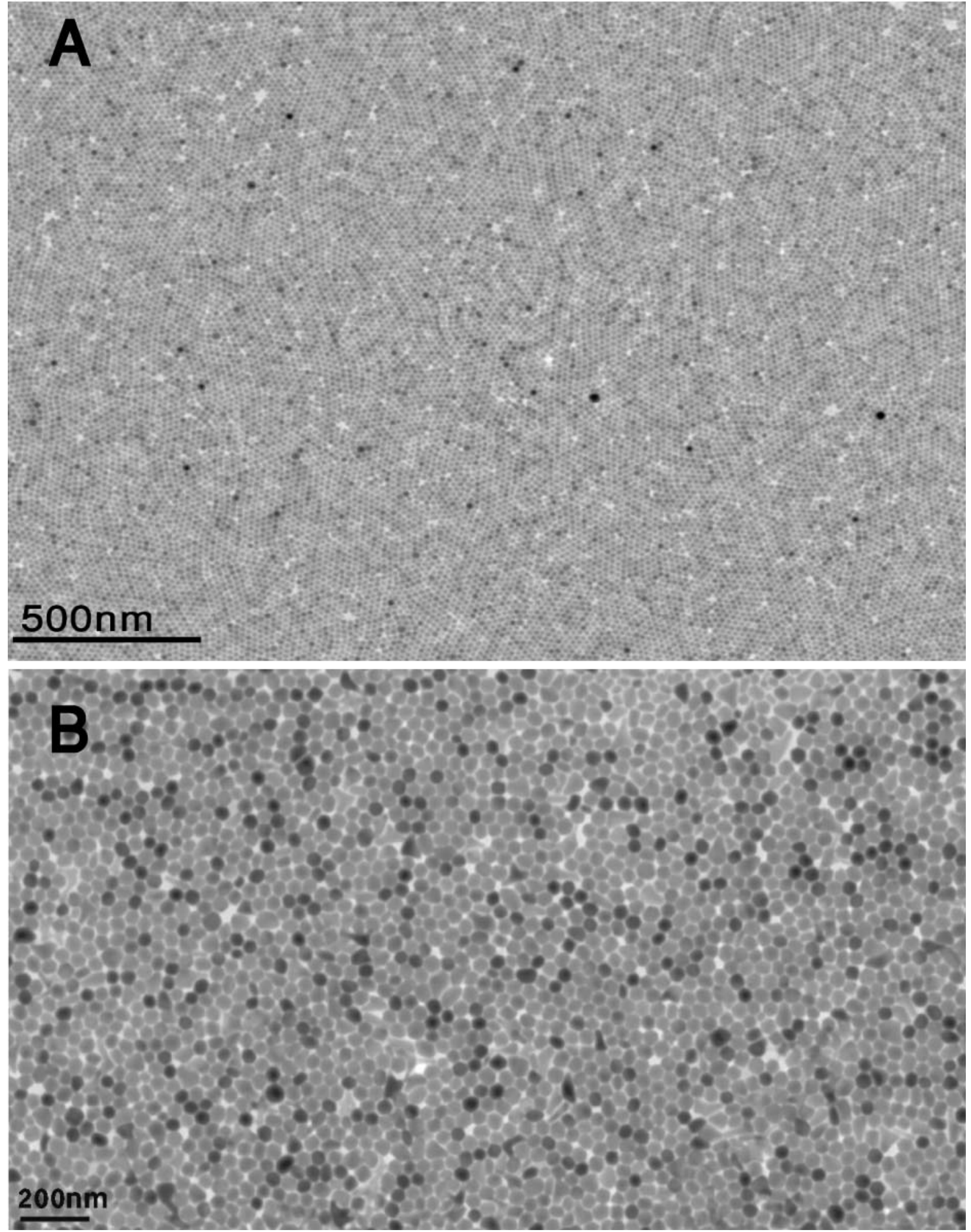

Fig. S4: LB films of closed packed PbTe nanocrystals, (A) cubes and (B) cuboctahedra. 